\title{
EXPECTATIONS OF SCATTER IN EQUIVALENT-DOSE DISTRIBUTIONS WHEN USING MULTI-GRAIN ALIQUOTS FOR OSL DATING
}

\author{
ALASTAIR C. CUNNINGHAM ${ }^{1}$, JAKOB WALLINGA ${ }^{1}$ \\ and PHILIP S.J. MINDERHOUD ${ }^{1,2}$ \\ ${ }^{1}$ Netherlands Centre for Luminescence dating, Delft University of Technology, Faculty of Applied Sciences, \\ Mekelweg 15, $2629 \mathrm{JB}$ Delft, the Netherlands \\ ${ }^{2}$ Department of Physical Geography, Utrecht University, Heidelberglaan 2, 3585 CS Utrecht, the Netherlands
}

Received 17 December 2010

Accepted 25 May 2011

\begin{abstract}
In the OSL dating of sediment, the scatter in equivalent dose $\left(D_{\mathrm{e}}\right)$ between grains is almost always larger than would be expected due to counting statistics alone. Some scatter may be caused by insufficient (partial) bleaching of some of the grains prior to deposition. In order to date partially bleached sediment, it is essential to estimate the amount of scatter caused by other processes (e.g. grain-to-grain variability in the natural dose rate). Measurements of such scatter are performed at the single-grain level; by contrast, most OSL dating is performed on multi-grain subsamples, for which grain-to-grain scatter is reduced through averaging.

Here we provide a model for estimating the expected scatter (i.e. excluding that caused by partial bleaching) for multi-grain aliquots. The model requires as input the single-grain sensitivity distribution, the number of grains in the sub-samples, and the expected scatter at the single-grain level, all of which can be estimated to an adequate degree. The model compares well with measured values of scatter in $D_{\mathrm{e}}$, determined using aliquots of various sizes, and can be used to help produce a minimumage $D_{\mathrm{e}}$ from multi-grain subsamples that is consistent with single-grain data.
\end{abstract}

Keywords: OSL, luminescence dating, overdispersion, minimum-age model, partial bleaching, aliquot size, MAM.

\section{INTRODUCTION}

Optically Stimulated Luminescence (OSL) dating of mineral grains requires an estimate of the radiation dose the grains have absorbed during the burial period. Commonly, the equivalent dose $\left(D_{\mathrm{e}}\right)$ is determined for several tens of aliquots, with each aliquot consisting of tens to thousands of grains. The $D_{\mathrm{e}}$ for each aliquot is estimated by comparing the 'natural' light yield from grains under optical stimulation, to the light yield observed under the

Corresponding author: A. C. Cunningham

e-mail: a.c.cunningham@tudelft.nl same conditions following one or more artificially given radiation doses (while also correcting for changes in sensitivity). Ideally, the $D_{\mathrm{e}}$ of each aliquot is found to be consistent with a common value (e.g. weighted average). However, $D_{\mathrm{e}}$ measurements frequently show a broader distribution than would be expected from counting statistics alone (e.g. Jacobs et al., 2008; Roberts et al., 1999).

An understanding of the sources of scatter is important for accurate age determination. This is particularly the case if it is suspected that the sediment contains grains that do not yield the desired burial dose information (e.g. OSL signal not reset in all grains prior to deposition and burial, post-depositional mixing of grains 
of different ages). For such situations, different statistical models have been introduced: Galbraith et al. (1999) have proposed the three and four parameter Minimum Age Models (MAM3, MAM4) to estimate the palaeodose from a $D_{\mathrm{e}}$ distribution which contains well bleached and partially or unbleached grains. Roberts et al. (2000) have provided a Finite Mixture Model (FMM) for the case where several distinct populations are present.

The MAM3, MAM4 and FMM require the prior determination of the amount of scatter that could be expected from a single, well-bleached population of grains. Referred to as 'overdispersion' $(\sigma)$, this term must incorporate every source of scatter except that from counting statistics and the scatter caused by the existence of more than one population (i.e. heterogeneous bleaching, mixing). Overdispersion is approximately the relative standard error of the underlying dose distribution (Galbraith et al., 1999).

Information on the causes of overdispersion in $D_{\mathrm{e}}$ distributions has been obtained from a number of studies using single-grain measurement systems (Thomsen et al., 2005; Duller et al., 2000) and/or modelling at the single grain level (Mayya et al., 2006; Nathan et al., 2003). It is likely that the majority of overdispersion comes from either micro-scale variations in natural dose rate, or from an as yet unexplained source of uncertainty in the $D_{\mathrm{e}}$ estimate observed for gamma-irradiated samples (Thomsen et al., 2005). Errors arising due to machine reproducibility typically make a small contribution to the overall uncertainty (e.g. $1.5 \%$ per OSL measurement for the Risø single-grain system (Thomsen et al., 2005)).

The MAM3 and FMM are designed for use with single-grain data (see e.g. Arnold and Roberts, 2009), and investigations on causes of overdispersion have also concentrated on single-grain data. By contrast, most OSL dating studies are carried out using small aliquots of quartz, with each aliquot containing tens, hundreds or thousands of grains. The use of multi-grain aliquots enables greater measurement precision and reduced measurement time; moreover, the apparatus required for single-grain measurements is not universally available. There is a need, therefore, of a means to convert information obtained from single-grain studies into a format applicable for OSL dating with multi-grain aliquots. Here we seek to address this issue, by establishing how the overdispersion parameter should be altered when the MAM3 is used with multi-grain aliquots. Furthermore, we consider an additional source of scatter relevant only to multi-grain aliquots, arising through the use of a nonhomogenous laboratory beta source.

\section{CORRECTION FOR ALIQUOT SIZE}

In single-grain dating studies of well-bleached quartz, the value of $\sigma$ is commonly found to be around $20 \%$ (Duller, 2008; Arnold and Roberts, 2009). However, when using aliquots with multiple grains, it can be ex- pected that $\sigma$ will be reduced, as a certain amount of averaging must occur. Failure to account for this effect when using the MAM3 will tend to lead to overestimation of $D_{\mathrm{e}}$, as the MAM3 will then allow for more overdispersion than is realistic. The extent of the averaging effect depends on the number of grains on the aliquot, and their respective intensities, and is determined here using stochastic simulations.

Since the advent of single-grain luminescencemeasurement technology (Bøtter-Jensen et al., 2000), it has become apparent that the OSL sensitivity of quartz varies dramatically between grains (Adamiec, 2000; Duller et al., 2000; Duller, 2008). Furthermore, the spread in grain sensitivity varies from sample to sample. With some highly sensitive samples, almost all quartz grains give a measurable OSL signal. More frequently however, it is only a small proportion of grains $(<5 \%)$ which provide most of the OSL signal. This variation can be seen in Fig. 1, which shows the cumulative distributions of single-grain OSL intensity for four samples discussed in this paper.

We consider here a sample of $n_{a}$ aliquots, with each aliquot containing $n_{g}$ grains. Each grain is assigned two values: Firstly, a value representing the grain-specific $D_{\mathrm{e}}$, which is drawn from a normal distribution with mean of zero and standard deviation of one. The normal distribution is used to simulate variation in $D_{\mathrm{e}}$ at the single grain level. The second value assigned to each grain is a sensitivity value, with each drawn randomly (with replacement) from the chosen dataset of single-grain sensitivity values. For each aliquot, we calculate the average $D_{\mathrm{e}}$, weighted by the sensitivity values, and the square root of the sum of the sensitivity values (which becomes the weight for the aliquot).

The statistic of interest is the standard deviation of $D_{\mathrm{e}}$ across $n_{a}$ aliquots. We chose $n_{a}=30$ in this simulation,

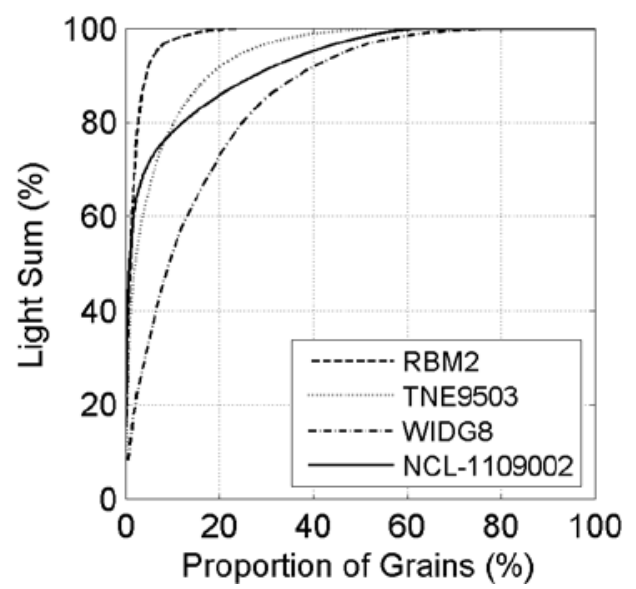

Fig. 1. The cumulative light sums for the four samples referred to in this paper, obtained through single-grain measurements. This figure reproduces Fig. 1 of Duller et al. (2000), with the addition of sample NCL-1109002. 
the number of aliquots typically measured for a single sample in dating applications. We use the weighted standard deviation $s$, taken from Galassi et al. (2009):

$$
s=\sqrt{\frac{1}{1-v} \sum_{i=1}^{n_{a}} w_{i}\left(x_{i}-\mu\right)^{2}}
$$

with normalized weights $w$ and weighted average $\mu$, and where:

$$
v=\sum_{i=1}^{n_{a}} w_{i}^{2}
$$

The use of $v$ of Eq. 2.1 accounts for the variability in luminescence sensitivity between aliquots. If there is little difference in sensitivity between aliquots, this term has little effect; on the other hand, a luminescence signal derived from a small percentage of the aliquots will lead to a larger $s$.

The results of this simulation are shown in Fig. 2, which plots the scatter in $D_{\mathrm{e}}$ as a function of the number of grains in each aliquot, for three different sensitivity distributions. The curves can be interpreted as the correction factor that should be applied to the single-grain overdispersion term when determining $\sigma$ for multi-grain studies. For example, if we were to measure $D_{\mathrm{e}}$ on 30 aliquots of sample RMB2 with 22 grains in each aliquot, and we have previously determined (or estimated) inherent scatter of $20 \%$ at the single grain level, then the $n_{g}$-corrected term is $20 \% \times 0.78=15.6 \%$ (this assumes that the measurement reproducibility errors have already been removed). As the sensitivity distribution of sample RMB2 is dominated by only a small percentage of the grains, and the number of grains on each aliquot is small, the reduction in the dispersion term is limited. For large aliquots of 1600 grains, the corrected term would be reduced to $20 \% \times 0.15=3 \%$. By contrast, if we repeat the calculations with sample WIDG8 (from which most grains give a significant luminescence signal), then we obtain $20 \% \times 0.40=8.0 \%$ and $20 \% \times 0.05=1 \%$ for aliquots containing 22 and 1800 grains, respectively. The correction of $\sigma$ creates an additional source of error, the size of which is dependent on the aliquot size and the number of aliquots used (Fig. 2).

It should be noted that while the averaging effect reduces scatter in $D_{\mathrm{e}}$ for larger aliquots, scatter caused by reproducibility error is not subject to the same relationship. The importance of reproducibility error therefore increases with the size of the aliquots, and should be accounted for separately (see section 5).

\section{ACCOUNTING FOR A NON-HOMOGENEOUS LABORATORY SOURCE}

An additional source of scatter relevant to multi-grain aliquots may come from variation in the dose rate provid-
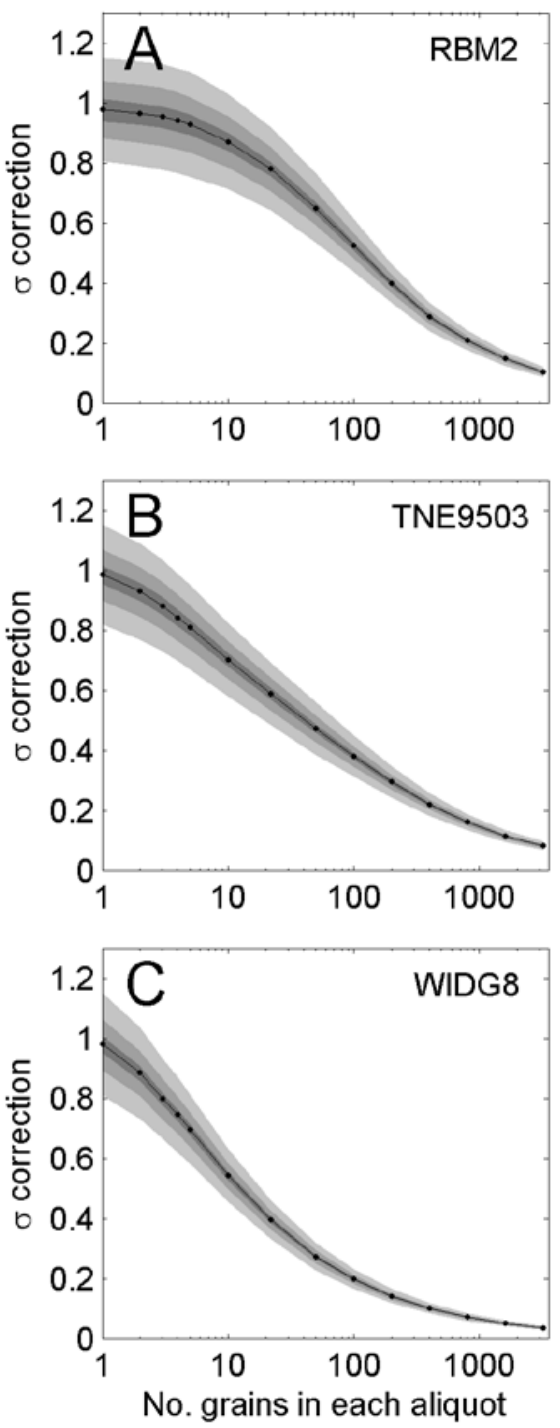

Fig. 2. The influence of aliquot size on overdispersion in $\mathrm{D}_{e}$, modelled using three different single-grain sensitivity distributions (RBM2, TNE9503, WIDG8, see Fig. 1). The y-axis term ' $\sigma$ correction' indicates the correction that must be made to the single-grain overdispersion term for use with multi-grain aliquots. The uncertainty on the correction depends on the number of aliquots $n_{a}$; standard error regions are shown for $n_{a}=30,10$, and 5 . If the correction is used with the MAM3, then the uncertainty is dependent on the number of aliquots consistent with the minimum age, not the total number of aliquots.

ed by the beta source. ${ }^{90} \mathrm{Sr} /{ }^{90} \mathrm{Y}$ beta sources are typically used to administer regenerative and test doses in OSL protocols. Inhomogeneity in a source may occur due to a number of reasons (see Ballarini et al. (2006) and references therein) and may lead to different grains receiving different regenerative doses. Under single-grain systems such source variability can be corrected for by grainspecific calibration, but this is not possible for multigrain aliquots, as it is not known which grains in an aliquot are producing the luminescence signal. Unlike other 
sources of measurement error, the effects of source variability will not be accounted for by a dose recovery test because the same source is used for both administering and estimating the 'given' dose.

To estimate the increase in scatter caused by an inhomogeneous source, we performed a similar simulation to that in section 2, but with the addition of grain-specific dose rates. The dose rates are calculated using the data of Ballarini et al. (2006), who showed an example of a nonhomogeneous laboratory source. The data of Ballarini et al. (2006) was created by measuring the OSL of calibration quartz on a 10 by 10 grid of single-grain holes, and is reproduced in Fig. 3. In our simulation, the laboratory dose rate for any position is calculated using a weighted average of the nearest measured points. The number of grains in each aliquot is a simple function of grain size and mask size, assuming $80 \%$ packing density (we use the term 'mask size' to indicate the diameter of the circular area on the disc containing the grains). Each grain is assigned two values: the laboratory dose rate determined by the position of each grain (randomly assigned within the area determined by the mask size); and a sensitivity value, drawn randomly, with replacement, from a specified dataset. We calculate the weighted-average laboratory dose rate for each aliquot. The dispersion caused by the laboratory dose-rate variability is then the standard deviation of the dose rate across $n_{a}$ aliquots (Eq. 2.1), divided by the mean laboratory dose rate. In this simulation, variation in $D_{\mathrm{e}}$ is not included.

The amount of additional dispersion caused by the non-homogeneous source is shown in Fig. 4 for three different grain sizes, and for each of the three single-grain

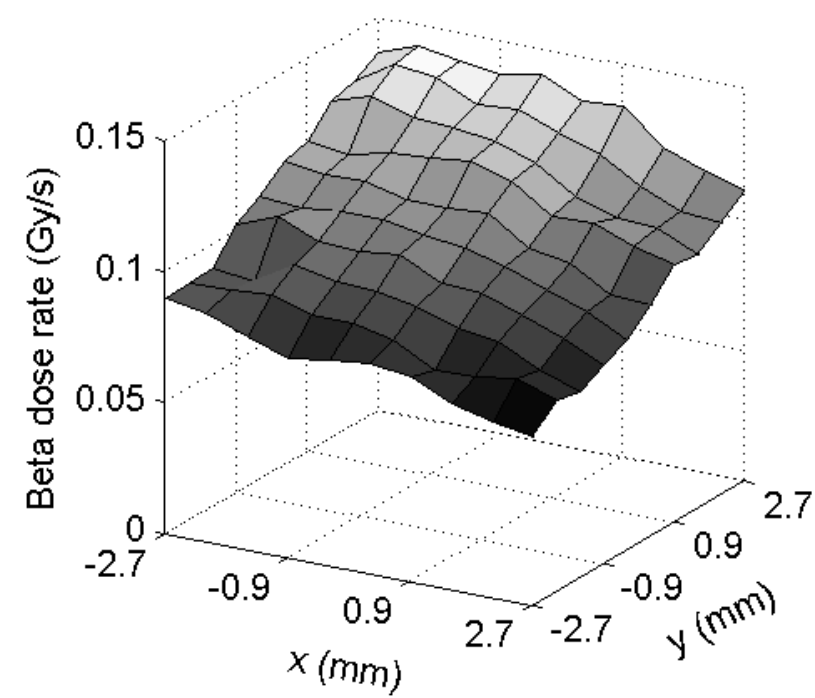

Fig. 3. Variation in dose rate across a single-grain disc, measured using calibration quartz on a $10 \times 10$ grid of single-grain holes. The minimum and maximum values are 0.064 and $0.142\left(\mathrm{~Gy} \mathrm{~s}^{-1}\right)$ respectively. The data is from Ballarini et al. (2006). datasets. The importance of grain size is in determining (with mask size) the number of grains on the aliquot. As the mask size increases, there are two competing effects: firstly, an increase in the range of laboratory dose rate applied to the grains; secondly, an increase in the averaging effect across the disc due to larger number of grains. The averaging effect is dominant for smaller grain sizes, larger mask sizes, and when the single-grain sensitivity distribution is more uniform (e.g. WIDG8).
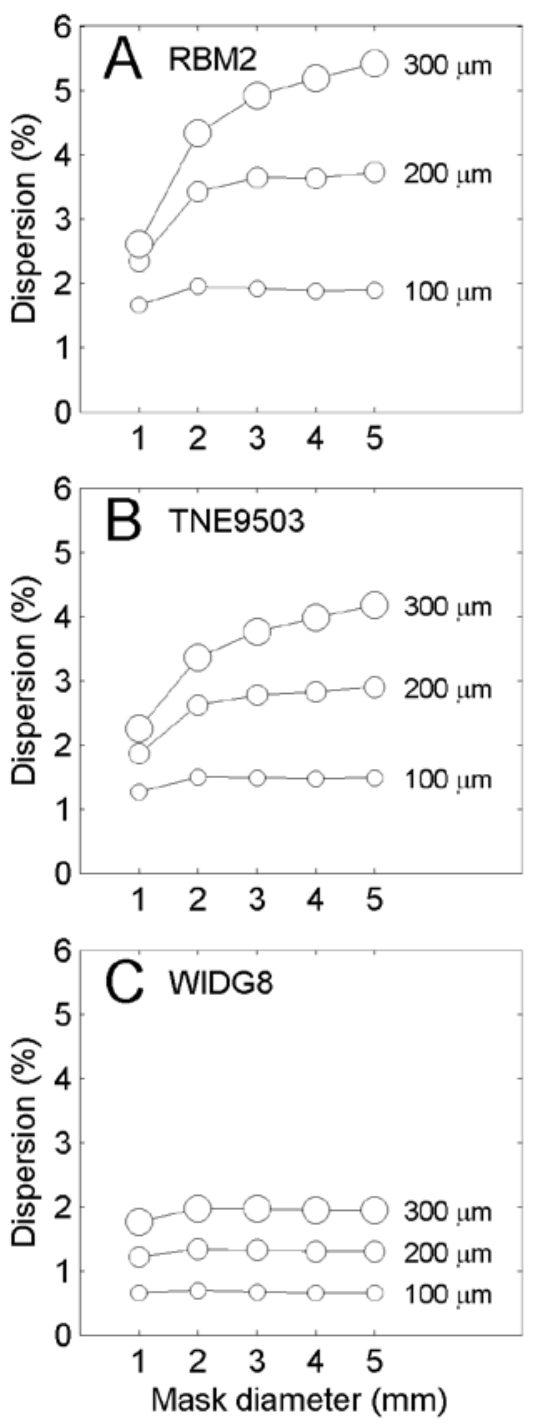

Fig. 4. Simulation of scatter in $\mathrm{D}_{e}$ due to a non-homogeneous beta source. Results depend on the mask size, grain size (which together determine the number of grains in the aliquot), and the single-grain sensitivity distribution (RBM2, TNE9503, WIDG8). 


\section{VALIDATION}

\section{Sample and measurement details}

The relationship between overdispersion and mask size is tested here using sample NCL-1109002, an aeolian coastal dune sample from the western Netherlands. The grain size of the sampled sediment is a relatively uniform 200-250 $\mu \mathrm{m}$. Quartz grains of 180-200 $\mu \mathrm{m}$ were extracted by sieving and chemical treatment $\left(\mathrm{HCl}, \mathrm{H}_{2} \mathrm{O}_{2}\right.$ and $\left.\mathrm{HF}\right)$.

Measurements were carried out on a Risø TL-DA-15 reader (Bøtter-Jensen et al., 2000), using a Single Aliquot Regenerative dose (SAR) protocol described in Table 1 (Murray and Wintle, 2000; 2003). Optical stimulation was with $470 \mathrm{~nm}$ diodes with a power of $\sim 35 \mathrm{~mW} \mathrm{~cm}^{-2}$ at the sample position. Irradiation was with a (homogenous) ${ }^{90} \mathrm{Sr} /{ }^{90} \mathrm{Y}$ beta source providing a dose rate of $\sim 0.13 \mathrm{~Gy} \mathrm{~s}{ }^{-1}$. Infrared (IR) diodes emitted at a wavelength of $875 \mathrm{~nm}$

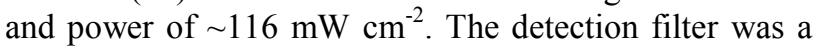
$7.5 \mathrm{~mm}$ Hoya U340. Single-grain measurements were made using a single-grain attachment to the reader, with stimulation by a Nd: $\mathrm{YVO}_{4}$ diode-pumped laser $(532 \mathrm{~nm}$ wavelength), with a $2.5 \mathrm{~mm}$ Hoya U340 detection filter (Ballarini et al., 2005).

Multi-grain aliquot OSL signals were processed using integration channels of $0-0.60 \mathrm{~s}$ for the initial signal, and $0.60-2.10 \mathrm{~s}$ for background subtraction. These intervals were selected in order to ensure that the net signal was dominated by the 'fast' OSL component, while keeping counting errors to acceptable levels (Cunningham and Wallinga, 2010). $D_{\mathrm{e}}$ for each aliquot was estimated using a linear fit to a single regenerative dose point (see Ballarini (2006) for a discussion on this point). This was followed by a 'zero' dose point, and two repeat points (the second following IR stimulation). Aliquots were accepted if recuperation was less than 0.05 Gy or was

Table 1. Details of the SAR protocol used

\begin{tabular}{ll}
\hline Treatment & Conditions \\
\hline Dose & $\mathrm{N}, 3.2,0,3.2 \mathrm{~Gy}$ \\
Preheat & $180^{\circ} \mathrm{C}$ for $10 \mathrm{~s}$ \\
OSL $(470 \mathrm{~nm})$ & $125^{\circ} \mathrm{C}$ for $40 \mathrm{~s}$ \\
Test dose & $3.2 \mathrm{~Gy}$ \\
Cutheat & $170^{\circ} \mathrm{C}$ \\
OSL $(470 \mathrm{~nm})$ & $125^{\circ} \mathrm{C}$ for $40 \mathrm{~s}$ \\
OSL Bleach $(470 \mathrm{~nm})$ & $180^{\circ} \mathrm{C}$ for $40 \mathrm{~s}$ \\
\hline
\end{tabular}

consistent with zero within the error term, and if the two recycling ratios were between 0.9 and 1.1 . For the singlegrain measurements, each grain was stimulated for $0.83 \mathrm{~s}$. We used the 'early background' principle for signal analysis, using the first $0.17 \mathrm{~s}$ for the initial signal, and the subsequent $0.42 \mathrm{~s}$ for background subtraction.

\section{Overdispersion as a function of aliquot size}

The dependence of overdispersion on aliquot size has been tested by measuring $D_{\mathrm{e}}$ using three different mask sizes: $2 \mathrm{~mm}$ ( $\sim 80$ grains), $3 \mathrm{~mm}$ ( $\sim 180$ grains) and $5 \mathrm{~mm}$ ( $\sim 500$ grains). The range of possible mask sizes is limited; below $2 \mathrm{~mm}$ there are too few grains in the aliquot to provide sufficient signal (for this sample); above $6 \mathrm{~mm}$ the grains on the edge of the mask area will receive a lower laboratory dose rate due to the geometry of the source (leading to larger $D_{\mathrm{e}}$ and more scatter). Results can be seen in Table 2 and Fig. 5.

As expected, the overdispersion in $D_{\mathrm{e}}$ decreases when the mask size increases. Unexpectedly, there is also a significant increase in $D_{\mathrm{e}}$ when the $5 \mathrm{~mm}$ mask size is used. It is possible that larger aliquots are more likely to include grains which are inappropriate for dating (e.g. feldspars, which are subject to a higher internal dose

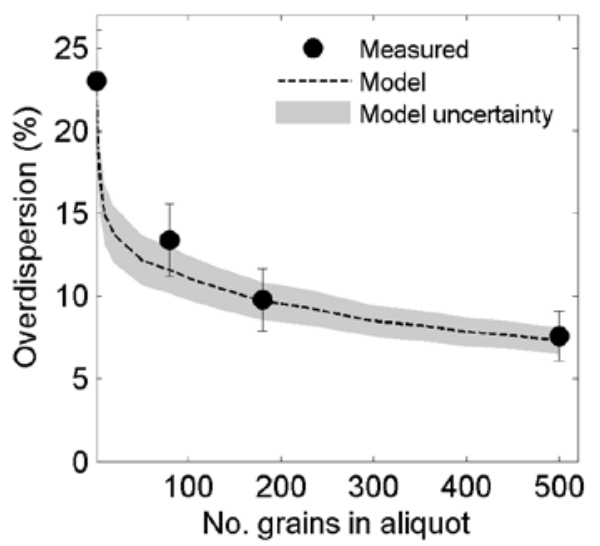

Fig. 5. Measured overdispersion $(\sigma)$ on $D_{e}$ for four different aliquot sizes of sample NCL-1109002, calculated using the Central Age Model of Galbraith et al. (1999). The model prediction is also shown, with the single-grain overdispersion $(23 \pm 3 \%)$ used as the basis of the model. Model uncertainty is based solely on the error term of the single-grain overdispersion measurement.

Table 2. Measurements of $\mathrm{D}_{e}$ and $\sigma$ for sample NCL-1109002, using different aliquot sizes, and results of dose recovery tests using different sources. The precise dose given with the gamma source is unknown.

\begin{tabular}{ccccc}
\hline Type & Mask & No. accepted & Given dose (Gy) & $D_{\mathrm{e}}(\mathrm{CAM})$ \\
\hline$D_{e}$ & Single grains & 31 & Natural & $1.150 \pm 0.049$ \\
$D_{e}$ & $2 \mathrm{~mm}(\sim 80$ grains $)$ & 37 & Natural & $1.068 \pm 0.029$ \\
$D_{e}$ & $3 \mathrm{~mm}(\sim 180$ grains $)$ & $27.0 \pm 3.1$ & $13.4 \pm 2.2$ \\
$D_{e}$ & $5 \mathrm{~mm}(\sim 500$ grains $)$ & 22 & Natural & $1.072 \pm 0.025$ \\
$9.8 \pm 1.9$ \\
Dose Recovery $(\beta)$ & $3 \mathrm{~mm}(\sim 180$ grains $)$ & 30 & Natural & $1.149 \pm 0.021$ \\
Dose Recovery $(\mathrm{y})$ & $3 \mathrm{~mm}(\sim 180$ grains $)$ & 35 & $?$ & $3.105 \pm 0.032$ \\
\hline
\end{tabular}


rate), but the strength of the OSL signal from other grains allows the aliquots to pass acceptance criteria. For smaller aliquots, such grains would be more likely to dominate the OSL signal, and to lead to rejection of the aliquot.

\section{Comparison with model}

We have applied the stochastic model described in section 2 to the sample used here. This calculation involves several steps. Firstly, the scatter caused by nonperfect measurement reproducibility was estimated, using a dose-recovery test. This test was carried out on 3-mm aliquots, using the built-in beta source to provide the dose. The $\sigma$ in the dose recovery results was found to be $3.0 \pm 1.2 \%$. Since this figure relates to machine uncertainty, we assume that it is identical for all aliquot sizes, including single grains. While it is likely that the additional complexity of the single-grain apparatus leads to less precision in measurement reproducibility, the extra dispersion (measured as $3.3 \%$ by Thomsen et al., 2005) is not significant when compared to the $\sim 20 \%$ spread in $D_{\mathrm{e}}$ observed at the single-grain level.

Using the stochastic model described in section 2, the expected relationship between $\sigma$ and aliquot size has been modelled, and is plotted in Fig. 5. The model uses several pieces of information: the single-grain sensitivity distribution for the sample, which was measured using a single-grain OSL reader (Fig. 1); the number of grains on the disc (determined by the mask size); the amount of scatter present at the grain-to-grain level (measured with single-grain OSL); and the scatter caused by machine reproducibility error $(3 \%)$. The measured single-grain overdispersion value of $23 \%$ is similar to previous studies: Arnold and Roberts (2009) have collated overdispersion data from published work, and found the mean overdispersion in single-grain studies of well-bleached samples to be $20 \%$, with a standard deviation of $9 \%$.

To check the source of the grain-to-grain scatter in $D_{\mathrm{e}}$, we conducted a further dose-recovery experiment. This time, the initial 'given' dose was administered using a separate ${ }^{60} \mathrm{Co}$ gamma source, which provided a uniform dose to the grains before they were placed on the stainless-steel discs (Bos et al., 2006). Using $3 \mathrm{~mm}$ aliquots, we found $\sigma$ of $9.7 \%$, far higher than the standard dose recovery of $3 \%$, and indistinguishable from $\sigma$ found on the natural sample $(9.8 \%)$. In other words, the precision with which we can recover a known dose is much poorer when that dose is given outside the measurement apparatus. A similar result was found by Thomsen et al. (2005), and indicates that the orientation of the grains with respect to the radiation field may be important, either through dose attenuation with depth in the grain, or some other effect.

\section{DISCUSSION}

\section{Aliquot size}

The use of larger aliquots leads to a reduction in interaliquot scatter in $D_{\mathrm{e}}$, and this process can be adequately described by the stochastic model presented above. Four pieces of information are required in order to use this model for a sample:

1) The number of grains in each aliquot.

2) The single-grain sensitivity distribution.

3) An estimate of $\sigma$ at the single-grain level

In order to use this model to generate the expectation of $\sigma$, each of these items of information needs to be estimated. The number of grains in each aliquot can be estimated by counting the grains on a selection of aliquots, or by calculating the number of grains that fit into the mask area. The single-grain sensitivity distribution, and the single-grain $\sigma$, will be unmeasured for most samples. However, good approximations could be made by using prior knowledge of similar samples, or with the data presented in this paper. In the absence of prior knowledge on sample characteristics, a good starting point would be to assume that the single-grain $\sigma$ is $20 \%$, and to pick a sensitivity distribution from Fig. 1 which seems most reasonable.

There is undoubtedly some uncertainty in the estimate of $\sigma$ for multi-grain aliquots. It should also be noted that the probability of the estimate being correct for a given selection of aliquots depends on the number of aliquots used (if using the CAM), or the number of aliquots from the well-bleached population (if using the MAM3).

To apply the model in practice, it is also necessary to estimate the uncertainty deriving from machine reproducibility (e.g. by using the CAM overdispersion term from dose-recovery data). As this sort of uncertainty does not get averaged out with more grains, it will tend to become more important for larger aliquots.

\section{Non-uniformity of the laboratory beta source}

It is encouraging that the averaging effect of large numbers of grains largely cancels out any nonhomogeneity in the laboratory beta source. This comes about for two reasons. Firstly, for the source used in our calculations, the gradient of the dose rate across the disc is relatively uniform, and the calculations assume the grains are equally likely to exist at any point within the mask area. The average dose rate is therefore largely independent of mask size, meaning that as the mask size is increased, the averaging effect caused by more grains overcomes the wider spread in laboratory dose rates between grains. Should either of these conditions not be met (e.g. a dome-shaped dose rate across the disc, or a nonuniform spread of grains across the mask area), then an increase in scatter in $D_{\mathrm{e}}$ would be observed with increasing mask size. 


\section{Example application: Estimating $\sigma$ for multi-grain aliquots}

We provide a brief example to demonstrate how the message of this paper can be used in practice. We use a sample of quartz grains from the banks of a stream in the Lushoto district, Tanzania (Sample code NCL-4211017). The grains are likely to have been deposited through fluvial and/or hillslope processes, and the data shows signs of insufficient bleaching. We carried out OSL measurements on small aliquots, with $2 \mathrm{~mm}$ mask size ( $\sim 80$ grains per aliquot, grainsize of $180-212 \mu \mathrm{m})$. The $D_{\mathrm{e}}$ distribution for the multi-grain aliquots is shown in Fig. 6a. To determine the burial dose using the MAM3, an appropriate value for $\sigma$ must be chosen. We start with the assumption that the true single-grain overdispersion in the burial dose is $20 \%$, and the single-grain sensitivity distribution approximates that of sample TNE9503 (i.e. a typical quartz sample; also found to be appropriate for this sample through single-grain sensitivity measurements). The expected $\sigma$ is then the sum of the following:

1) Grain-to-grain scatter, corrected for the number of grains on the disc. From Fig. 2b, the correction is $\sim 0.40$; the corrected overdispersion term is then $0.20 \times 0.40=0.08$

2) Measurement reproducibility errors: we use $3 \%$, taken from the measured overdispersion of the doserecovery data (Table 2 ).

Added in quadrature, the best estimate for $\sigma$ is 0.085 . To determine the minimum-age $D_{\mathrm{e}}$ for this sample, the $\sigma$ value can be added (in quadrature) to the individual $D_{\mathrm{e}}$ error terms before using the MAM3. The outcome of this process is a minimum-age $D_{\mathrm{e}}$ of $0.306 \pm 0.025 \mathrm{~Gy}$ (single point shown in Fig. 6c). Fig. $6 c$ also shows the dependence of the mimimum-age $D_{\mathrm{e}}$ on the chosen value of $\sigma$; for this sample, there is relatively little change in $D_{\mathrm{e}}$ for $\sigma$ between 0 and 0.15 , but a significant shift in $D_{\mathrm{e}}$ for higher $\sigma$ values. To validate the multi-grain aliquot approach, we also show results from single-grain $D_{\mathrm{e}}$ measurements of the same sample (Fig. 6b). Assuming a $\sigma$ of 0.20 for the single grain data provides a minimum-age $D_{\mathrm{e}}$ of $0.302 \pm$ $0.018 \mathrm{~Gy}$, indistinguishable from the multi-grain aliquot estimate.

\section{CONCLUSION}

Use of the minimum age model for partially bleached (or mixed) samples requires an estimate of $\sigma$, the amount of scatter in $D_{\mathrm{e}}$ from sources other than partial bleaching (or mixing) and counting statistics. This scatter is present at the single-grain level, but with multi-grain aliquots the effect is reduced because of averaging in each aliquot. This process can be described by the stochastic model presented here, which requires the following information:

1) An estimate of overdispersion at the single-grain level.
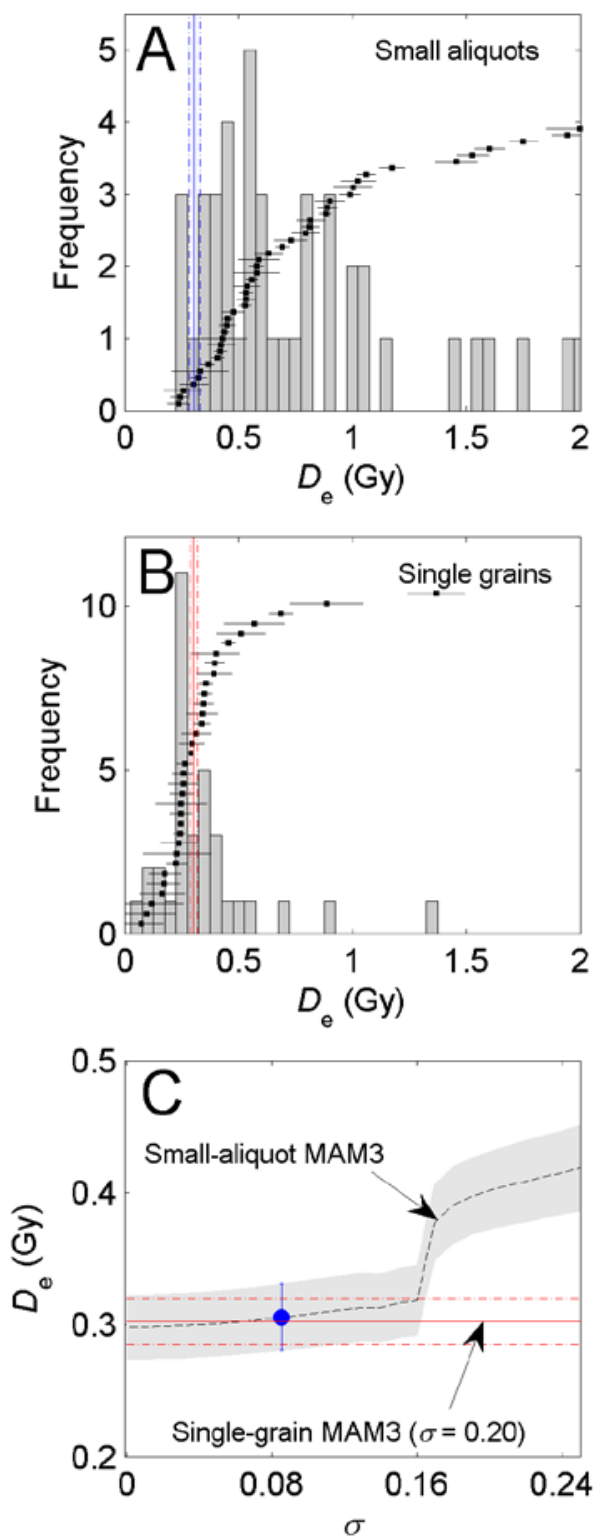

Fig. 6. (a) Histogram of $\mathrm{D}_{e}$ for sample NCL-411017 (Tanzania), measured using aliquots of $\sim 80$ grains each. 54 aliquots passed acceptance criteria (recuperation less than $10 \%$ of regenerative dose, both recycling ratios between 0.9 and 1.1); There are 11 aliquots giving $D_{e}$ greater than $2 \mathrm{~Gy}$ which are not shown. Using $\sigma_{b}$ of 0.085 , the MAM3 $\mathrm{D}_{e}$ is $0.306 \pm 0.025$, shown by vertical lines in the figure. (b) Histogram of single-grain $\mathrm{D}_{e}$ values for the same sample; MAM3 gives $0.302 \pm$ $0.018 \mathrm{~Gy}\left(\sigma_{b}=0.20\right.$; one imprecise negative- $\mathrm{D}_{e}$ grain was excluded). (c) The dependence of the multi-grain-aliquot MAM3 $\mathrm{D}_{e}$ on the specified $\sigma_{b}$ value for this sample. The suggested $\sigma_{b}$ value of 0.085 (single data-point) provides a minimum age in agreement with that derived from the single-grain data (horizontal lines). Measurement details are the same as those described in section 4, other the preheat and cutheat temperatures $\left(240^{\circ} \mathrm{C}\right.$ and $220^{\circ} \mathrm{C}$, respectively, with a hightemperature bleach of $250^{\circ} \mathrm{C}$ ). 
2) The number of grains in each aliquot.

3) The single-grain sensitivity distribution.

4) The machine/measurement uncertainty

A similar model can be used to estimate the increase in scatter caused by a non-homogeneous laboratory betasource. For the particular beta-source described in this paper, with dose rates differing by a factor two across the disc, the additional scatter was found to be largely insignificant compared with other sources of scatter for aliquots up to $5 \mathrm{~mm}$ diameter.

\section{ACKNOWLEDGEMENTS}

The authors are supported by NWO/STW grant DSF.7553. The scripts were written in Matlab, and are available from AC. We thank Geoff Duller for providing the information on sensitivity distributions, and Mirko Ballarini for providing the dataset on source inhomogeneity. The manuscript was improved following comments from two anonymous referees.

\section{REFERENCES}

Adamiec G, 2000. Variations in luminescence properties of single quartz grains and their consequences for equivalent dose estimation. Radiation Measurements 32(5-6):427-432, DOI 10.1016/S1350-4487(00)00043-3.

Arnold LJ and Roberts RG, 2009. Stochastic modelling of multi-grain equivalent dose (De) distributions: Implications for OSL dating of sediment mixtures. Quaternary Geochronology 4(3): 204-230, DOI 10.1016/j.quageo.2008.12.001.

Ballarini M, 2006. Optical dating of quartz from young deposits. PhD thesis, Delft University of Technology

Ballarini M, Wallinga J, Duller GAT, Brower JC, Bos AJJ and van Eijk CWE, 2005. Optimuzing detection filters for single grain optical dating of quartz. Radiation Measurements 40(1): 5-12, DOI 10.1016/j.radmeas.2005.03.006.

Ballarini M, Wintle AG and Wallinga J, 2006. Spatial variation of dose rate from beta sources using single grains. Ancient TL 24(1):1-8.

Bos AJJ, Wallinga J, Johns C, Abellon RD, Brouwer JC, Schaart DR and Murray AS, 2006. Accurate calibration of a laboratory beta particle dose rate for dating purposes. Radiation Measurements 41(7-8): 1020-1025, DOI 10.1016/j.radmeas.2006.04.003.

Bøtter-Jensen L, Bulur E, Duller GAT and Murray AS, 2000. Advances in luminescence instrument systems. Radiation Measurements 32(1): 57-73, DOI 10.1016/S1350-4487(99)00253-X.

Cunningham AC and Wallinga J, 2010. Selection of integration time- intervals for quartz OSL decay curves. Quaternary Geochronology 5(6): 657-666, DOI 10.1016/j.quageo.2010.08.004.

Duller GAT, 2008. Single-grain optical dating of Quaternary sediments: why aliquot size matters in luminescence dating. Boreas 37(4): 589-612, DOI 10.1111/j.1502-3885.2008.00051.x.

Duller GAT, Bøtter-Jensen L and Murray AS, 2000. Optical dating of single sand-sized grains of quartz: sources of variability. Radiation Measurements 32(5-6): 453-457, DOI 10.1016/S13504487(00)00055-X.

Galassi M, Davies J, Theiler J, Gough B, Jungman G, Alken P, Booth $\mathrm{M}$ and Rossi F, 2009. GNU Scientific Library Reference Manual. Network Theory Ltd.

Galbraith RF, Roberts RG, Laslett GM, Yoshida H and Olley JM, 1999. Optical dating of single and multiple grains of quartz from jinmium rock shelter, northern Australia, part 1, Experimental design and statistical models. Archaeometry 41(2): 339-364, DOI 10.1111/j.1475-4754.1999.tb00987.x.

Jacobs Z, Wintle AG, Roberts RG and Duller GAT, 2008. Equivalent dose distributions from single grains of quartz at Sibudu, South Africa: context, causes and consequences for optical dating of archaeological deposits. Journal of Archaeological Science 35(7): 1808-1820, DOI 10.1016/j.jas.2007.11.027.

Mayya YS, Morthekai P, Murari MK and Singhvi AK, 2006. Towards quantifying beta microdosimetric effects in single-grain quartz dose distribution. Radiation Measurements 41(7-8): 1032-1039, DOI 10.1016/j.radmeas.2006.08.004.

Murray AS and Wintle AG, 2000. Luminescence dating of quartz using an improved single-aliquot regenerative-dose protocol. Radiation Measurements 32(1): 57-73, DOI 10.1016/S1350-4487(99)00253$\mathrm{X}$.

Murray AS and Wintle AG, 2003. The single aliquot regenerative dose protocol: potential for improvements in reliability. Radiation Measurements 37(4-5): $\quad 377-381$, DOI $\quad 10.1016 / \mathrm{S} 1350$ 4487(03)00053-2.

Nathan RP, Thomas PJ, Jain M, Murray AS and Rhodes EJ, 2003. Environmental dose rate heterogeneityof beta radiation and its implications for luminescence dating: Monte Carlo modeling and experimental validation. Radiation Measurements 37(4-5): 305-313, DOI 10.1016/S1350-4487(03)00008-8.

Roberts RG, Galbraith RF, Olley JM, Yoshida H and Laslett GM, 1999. Optical dating of single and multiple grains of quartz from Jinmium rock shelter, northern Australia: part II, results and implications. Archaeometry 41(2): 365-395, DOI 10.1111/j.14754754.1999.tb00988.x.

Roberts RG, Galbraith RF, Yoshida H, Laslett GM and Olley JM, 2000. Distinguishing dose populations in sediment mixtures: a test of single-grain optical dating procedures using mixturesof laboratorydosed quartz. Radiation Measurements 32(5-6): 459-465, DOI 10.1016/S1350-4487(00)00104-9.

Thomsen KJ, Murray AS and Bøtter-Jensen L, 2005. Sources of variability in OSL dose measurements using single grains of quartz. Radiation Measurements 39(1): 47-61, DOI 10.1016/j.radmeas.2004.01.039. 